\title{
A viral protein that selectively downregulates ICAM-1 and B7-2 and modulates $T$ cell costimulation
}

\author{
Laurent Coscoy and Don Ganem
}

Howard Hughes Medical Institute and Departments of Microbiology and Medicine, University of California Medical Center, San Francisco, California, USA

Address correspondence to: Don Ganem, University of California Medical Center, San Francisco, California 94143-0414, USA. Phone: (415) 476-2826; Fax: (415) 476-0939; E-mail: ganem@cgl.ucsf.edu.

Received for publication February 2, 2001, and accepted in revised form May 14, 2001.

\begin{abstract}
Kaposi's sarcoma-associated (KS-associated) herpesvirus (KSHV) is a B-lymphotropic agent linked to AIDS-related lymphoproliferative disorders and KS. We and others have earlier identified two viral genes, $K 3$ and $K 5$, that encode endoplasmic reticulum proteins that downregulate surface MHC-I chains by enhancing their endocytosis. Here we have examined the ability of these proteins to influence the disposition of other host surface proteins implicated in immune recognition and activation. We report that $\mathrm{K} 5$, but not $\mathrm{K} 3$, expression in $\mathrm{BJAB}$ cells dramatically reduces ICAM-1 and B7-2 surface expression; B7-1 expression is unaffected. This $\mathrm{K} 5$-induced reduction can be reversed by coexpression of a dominant negative mutant of dynamin, indicating that the loss of ICAM and B7-2 surface expression is due to their enhanced endocytosis. This downregulation is functionally significant, because K5-transfected B cells show substantial impairment in their ability to induce T cell activation. $\mathrm{K} 5$ is thus the first example of a viral modulator of immunological synapse formation and $\mathrm{T}$ cell costimulation. We propose that its expression reduces $\mathrm{T}$ cell responses to KSHVinfected B cells early in infection, thereby diminishing antiviral cytokine release and the production of stimulatory signals for CTL generation.
\end{abstract}

J. Clin. Invest. 107:1599-1606 (2001).

\section{Introduction}

The generation of an adaptive immune response is a complex process that requires the engagement of $\mathrm{T}$ cells with professional antigen-presenting cells (APCs), including dendritic cells, macrophages, and B cells. A key step in this process is the activation of the T cell, mediated by interaction of the $\mathrm{T}$ cell antigen receptor (TCR) with antigenic peptides presented by MHC molecules on the APCs (1). Sustained TCR engagement is required to generate the full repertoire of $\mathrm{T}$ cell responses, including signal transduction, cytokine generation, and cell proliferation $(2,3)$. Although the TCRpeptide-MHC interaction affords the requisite specificity for such responses, it does not suffice to ensure the sustained engagement needed for effective activation. First, this interaction is generally of modest affinity, and the number of antigenic complexes on the APC surface can be low (4-8). Second, the presence of abundant and sizeable glycoproteins (e.g., the mucins CD43 and CD45) on cell surfaces poses additional barriers to effective cell-cell contact $(9,10)$. Finally, there is strong evidence that signaling from the TCR receptor is insufficient for $\mathrm{T}$ cell activation (2); costimulatory signals are required from accessory receptors, most notably CD28, by interaction with its ligands B7-1 and B7-2 on the APC surface (11-15). The interaction of LFA-1 with its ligand ICAM-1 is also well known to be an important participant in the activation of T cells (16-18), lowering the concentration of antigen required for stimulation and promoting more sustained signaling from the TCR. Accordingly, APCs expressing both ICAM-1 and B7 isoforms display enhanced stimulation of T cell proliferation and IL-2 secretion (19-21).

The recognition and signaling events just described are now known to be effected by a dynamic structure known as the immunological synapse, which is assembled upon T cell-APC contact (22-24). The dynamics of this assembly process are beginning to be understood. In addition to TCR-MHC recognition, adhesion molecules on the APC, notably ICAM-1, make contact with their ligands (for ICAM-1, LFA-1) on the $\mathrm{T}$ cell and promote apposition of the cell surfaces. This initiates an active process of cytoskeletal and membrane reorganization that culminates in the generation of an ordered structure in which a central core bearing the TCR-MHC partners is ringed by ICAM/LFA complexes (25-27). This rearrangement contemporaneously recruits to the synapse-specialized membrane lipid microdomains (rafts) bearing associated signaling components (kinases, adaptor proteins). The net result is a structure designed to assemble and concentrate all the components needed for effective $T$ cell activation.

A prime function of the adaptive immune response is defense against exogenous pathogens. Viruses and in particular herpesviruses - have in turn evolved elaborate strategies for evading such responses (28). Most of these have revolved around down- 

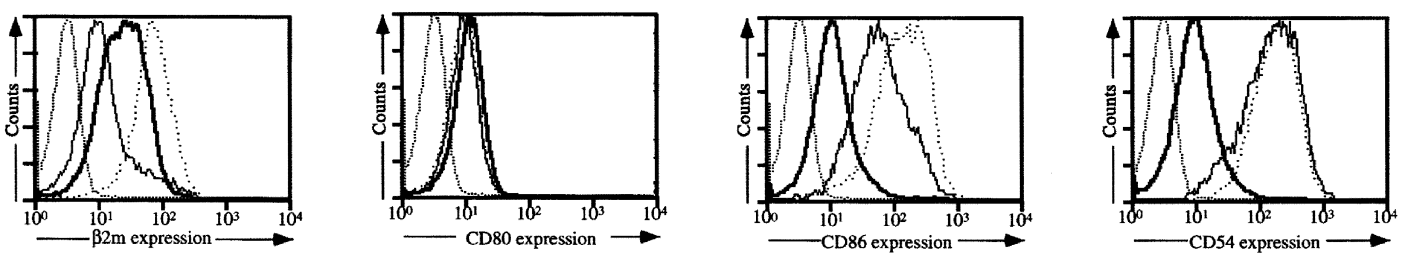

.............. BJAB-vector

- BJAB-K5
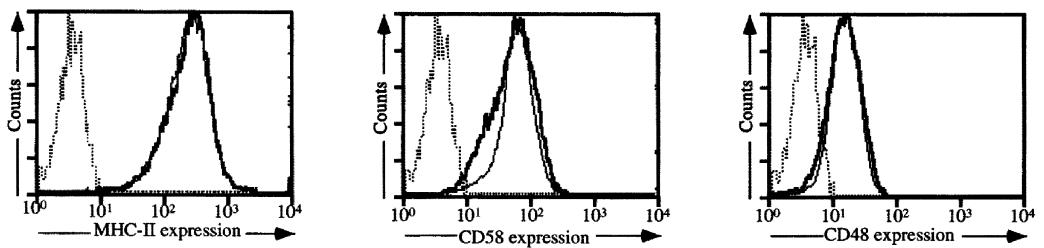

-.......... secondary only
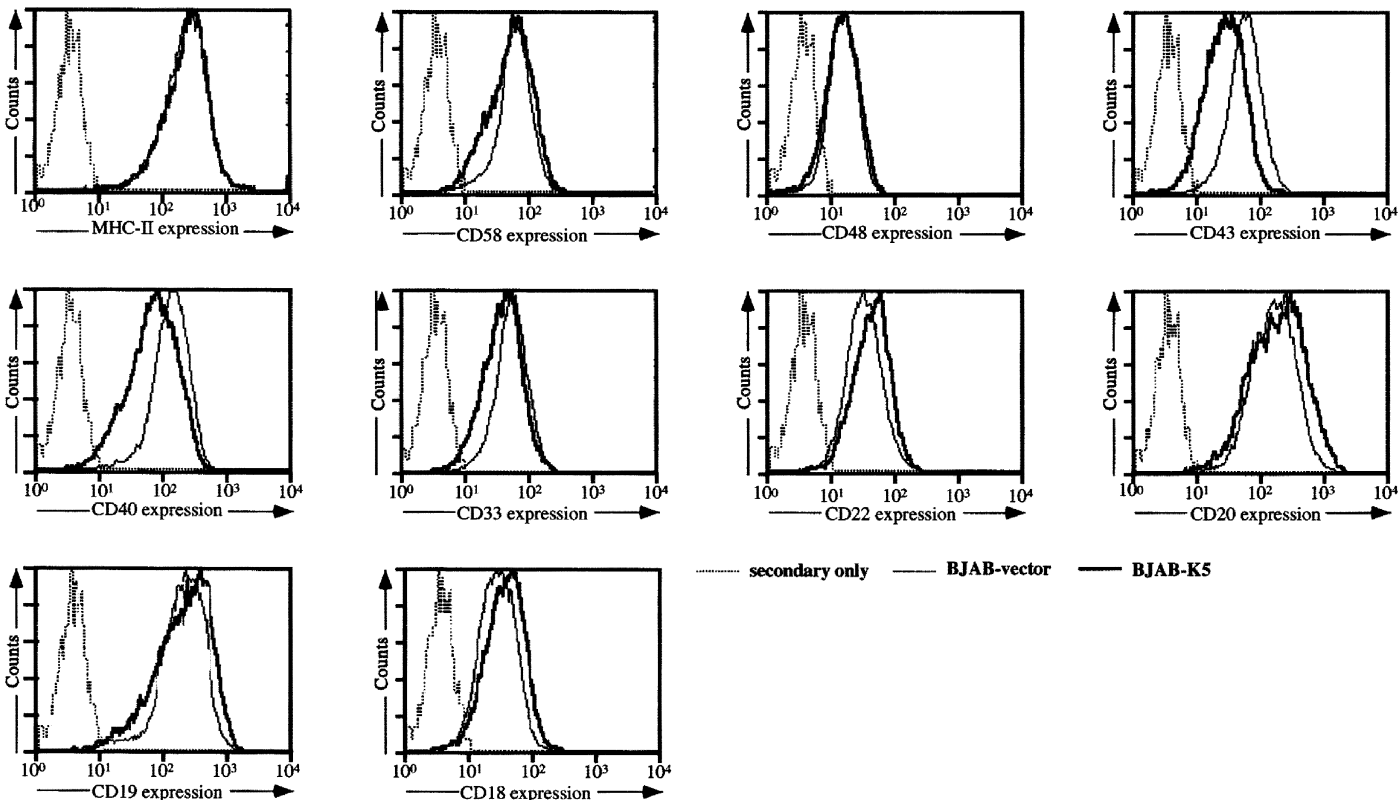

B......... secondary only __ BJAB-vector

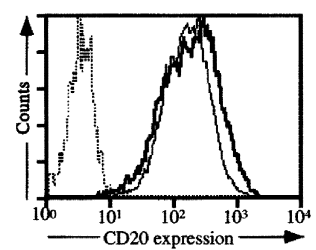

- BJAB-K5

\section{Figure 1}

Surface expression of molecules associated with T cell activation. BJAB cells were transduced with PMX-pie (as control), PMX-FlagK5, or PMX-HaK3 retroviral vectors. Cells were stained with mAb's directed against $B$ cell membrane proteins associated with $T$ cell activation (MHC-II, B7-2, B7-1, ICAM-1, CD58, CD45, and CD43) as well as other B cell membrane proteins as control (CD48, CD40, CD33, CD22, CD20, CD19, CD18, $\beta 2 \mathrm{~m})$. Bound Ab's were revealed by an R-phycoerythrin-conjugated goat anti-mouse Ab, and fluorescence intensity was monitored by flow cytometry. WT, wild-type.

regulation of surface MHC-I display, allowing them to avoid cytotoxic $\mathrm{T}$ lymphocyte (CTL) recognition (29-32). Recently, we (33) and others $(34,35)$ have identified two novel proteins that mediate MHC-I downregulation in the Kaposi's sarcoma-associated herpesvirus (KSHV) (also called human herpesvirus8). KSHV is a lymphotropic herpesvirus; infection by this agent is strongly linked to both Kaposi's sarcoma and several AIDS-associated lymphoproliferative syndromes: primary effusion lymphoma (PEL) and multicentric Castleman's disease (36). These proteins, encoded by KSHV genes $K 3$ and $K 5$, are unrelated to other viral or cellular proteins, but share $40 \%$ amino acid sequence identity with one another. They are localized to the endoplasmic reticulum (ER) but mediate their action distally in the secretory pathway, promoting endocytosis of surface MHC-I chains. This MHC-I downregulation has also been observed recently in cultured PEL cell lines undergoing lytic reactivation of KSHV (37). Because KSHV predominantly infects B lymphocytes $(36,38)$, a cell type competent for antigen presentation to $\mathrm{T}$ cells, we hypoth- esized that this virus may also encode proteins that specifically alter $\mathrm{T}$ cell activation. Accordingly, we tested the ability of K3 and K5 to modulate the surface display of components involved in the formation or function of the immunological synapse.

\section{Methods}

Plasmids. A Ha-tagged version of K3 and Flag-tagged version of $\mathrm{K} 5$ were subcloned from $\mathrm{PCR} 3.1-\mathrm{HaK} 3$ and pCR3.1-HaK3 into PMX-pie retroviral vector (kindly provided by L. Lanier, University of California at San Francisco, San Francisco, California, USA). PMX-pie contains an internal ribosome entry site (IRES) between the multiple cloning site (MCS) and the enhanced green fluorescent protein (EGFP). This permits both the gene of interest (cloned into the MCS) and the EGFP to be translated from a single bicistronic mRNA transcribed from the viral longterminal repeat. In addition, PMX-pie expresses the puromycin resistance gene from a SV40 promoter. The Flag-tagged version of the K5 gene was subcloned from pCR3.1-FlagK5 vector into pBMN-ZIN 
(kindly provided by G. Nolan, Stanford University). This retroviral vector permits the expression of FlagK5 and the neomycin resistance gene from a single bicistronic mRNA. Vectors expressing the wildtype dynamin or the K44E dynamin-1 transdominant negative were kindly provided by R. Vallee (University of Massachusetts Medical School). Plasmids encoding the luciferase gene under the control of $\mathrm{RE} / \mathrm{AP}, \mathrm{NFAT}, \mathrm{AP}-1$, or NF- $\mathrm{KB}$ transcription elements were kindly provided by J. Shapiro (University of California at San Francisco).

$A b$ 's and reagents. For FACS analysis all $A b$ 's were used at a concentration of $1 \mu \mathrm{g} / 10^{6}$ cells. Anti-CD 18 , -CD33, -CD43, -CD45, and -CD48 were kindly provided by L. Lanier (University of California at San Francisco). Anti-CD19, -CD20, -CD22,-CD40, -CD58, -ICAM-1, -B7-1, -B7-2,-MHC-II, and - $\beta 2 \mathrm{~m}$ were purchased from PharMingen (San Diego, California, USA). Anti-Ha tag fluorescein-conjugated $\mathrm{mAb}$ 's were purchased from Santa Cruz Biotechnology Inc. (Santa Cruz, California, USA). Staphylococcal enterotoxin E (SEE) was purchased from Toxin Technology Inc. (Sarasota, Florida, USA). Ammonium chloride (Fisher Scientific Co., Fair Lawn, New Jersey, USA) was used at $50 \mathrm{mM}$. Tetradecanoyl phorbol acetate (TPA) (Calbiochem-Novabiochem Corp., La Jolla, California, USA) was used at $20 \mathrm{ng} / \mathrm{ml}$, and ionomycin (Sigma Chemical Co., St. Louis, Missouri, USA) was used at 500 nM. Fugene6 was from Roche Diagnostics Corp. (Indianapolis, Indiana, USA) and used according to the manufacturer's recommendation.

Cell lines. BJAB, 721.221, and Jurkat cells were grown in RPMI-1640 medium supplemented with 10\% FCS and penicillin-streptomycin. The NKL cell line was grown in RPMI-1640 medium supplemented with 10\% FCS, penicillin-streptomycin, none essential amino acids, $5.5 \times 10^{2}$ $\mathrm{mM}$ 2-mercaptoethanol, $10 \mathrm{mM}$ HEPES, $2 \mathrm{mM}$ L-glutamine, $1 \mathrm{mM}$ sodium pyruvate, and $200 \mathrm{U} / \mathrm{ml}$ of human recombinant IL-2 (Roche Diagnostics Corp.).

Transfections and luciferase assays. Cells were washed and resuspended at $2 \times 10^{7}$ cells $/ \mathrm{ml}$. A total of $450 \mu \mathrm{l}$ of cells and $20 \mu \mathrm{g}$ of DNA were electroporated in a $0.4-\mathrm{cm}$ gap cuvette at $250 \mathrm{~V}, 960 \mu \mathrm{F}$, using a BioRad gene pulser (Bio-Rad Laboratories Inc., Richmond, California, USA). For luciferase assays, cells were lysed directly into the wells using $30 \mu \mathrm{l}$ of $5 \times$ reporter lysis buffer (Promega Corp., Madison, Wisconsin, USA). Fifty microliters of extract were then used for quantification using a DLReady (Turner Designs Inc., Sunnyvale, California, USA) or an Optocomp-1 (MGM Instruments Inc., Hamden, Connecticut, USA) luminometer.

Retroviral infection. On transfection with retroviral vectors (PMX-pie or $\mathrm{pBMN}$ ), the Phoenix packaging cell line produces replication-defective viral particles that can be used for stable expression in BJAB cells. Phoenix cells were transfected using Fugene6 according to the manufacturer's recommendation. Viruscontaining supernatant was harvested 48 hours after transfection, filtered through a $0.45-\mu \mathrm{m}$ filter and
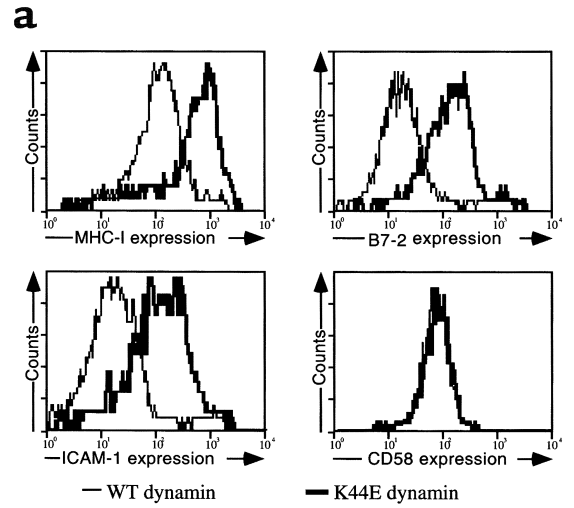

b
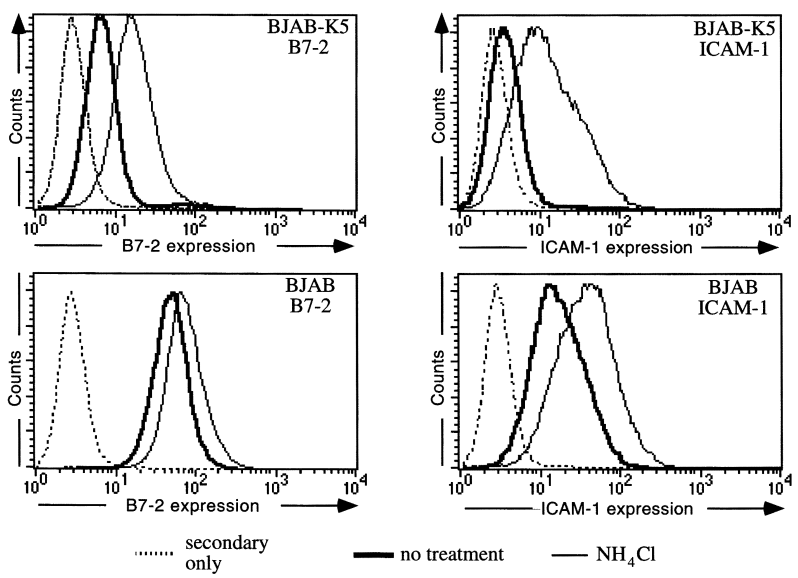

Figure 2

(a) Endocytosis of B cell membrane proteins in $\mathrm{K} 5$ expressing $\mathrm{BJ} A \mathrm{~B}$. $B J A B$ cells expressing $K 5$ were transfected with $20 \mu \mathrm{g}$ of an expression vector for a Ha-tagged dynamin transdominant mutant (known to specifically block endocytosis events) or for an expression vector for the Ha-tagged wild-type dynamin (as control). Forty-eight hours after transfection, cells were incubated with $m A b$ directed against either MHC-I, B7-2, ICAM-1, or CD58 and bound mAb's were revealed by incubation with an R-phycoerythrin-conjugated goat anti-mouse $A b$. Cells were then fixed, incubated in presence of saponin with a fluorescein-conjugated $\mathrm{mAb}$ directed against the $\mathrm{Ha}$ tag, and analyzed by flow cytometry. Results represent the cell surface expression of MHC-I, B7-2, ICAM-1, and CD58 proteins in the dynamin-transfected cells. (b) BJAB cells expressing $\mathrm{K} 5$ or control cells were incubated for 12 hours in presence of $50 \mathrm{mM}$ ammonium chloride. Cells were then fixed, incubated with mAb's against B7-2 and ICAM- 1 in the presence of saponin, and analyzed by flow cytometry.

diluted with Polybrene $(4 \mu \mathrm{g} / \mathrm{ml}$ final dilution). BJAB cells $\left(5 \times 10^{6}\right)$ were infected by spin infection $(800 \mathrm{~g}$ for 2 hours at $20^{\circ} \mathrm{C}$ ) using $2 \mathrm{ml}$ of viral supernatant. Selection of transduced BJAB was started 36 hours after infection by adding $1.5 \mathrm{mg} / \mathrm{ml}$ of G418 or 1 $\mu \mathrm{g} / \mathrm{ml}$ of puromycin.

Flow cytometry analysis. Cells were washed in PBS 1\% BSA and incubated with specific mAb's $\left(1 \mu \mathrm{g} / 10^{6}\right.$ cell $)$ for 30 minutes at $4^{\circ} \mathrm{C}$. Bound mouse $\mathrm{Ab}$ 's were revealed by an R-phycoerythrin-conjugated goat antimouse Ab. Cell surface fluorescence was analyzed with a Becton Dickinson FACScalibur (Becton Dickinson, San Jose, California, USA). For intracellular staining, 


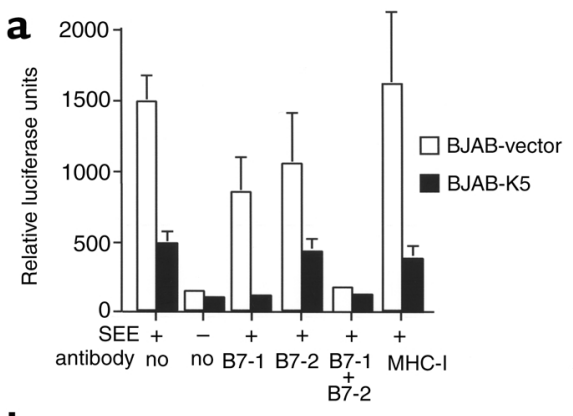

b

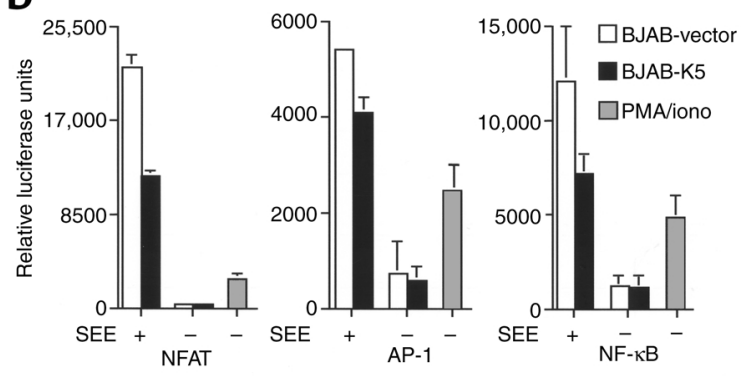

c

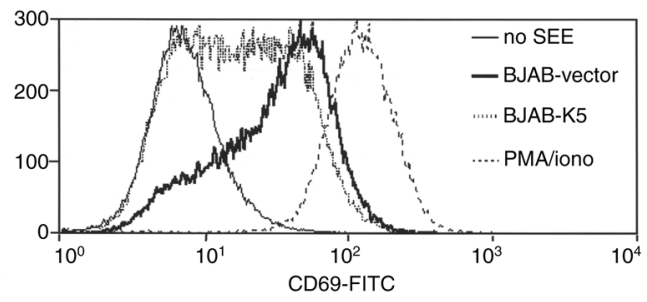

Figure 3

$\mathrm{T}$ cell activation by $\mathrm{K} 5$ expressing BJAB cells. (a) Twenty-four hours after transfection with a RE/AP reporter plasmid, Jurkat cells were stimulated by incubation with BJAB cells and SEE. Jurkat activation was quantified by luciferase assays 16 hours after stimulation. Each luciferase assay was done in triplicate on at least three different independent transfection experiments. For blocking conditions, BJAB cells were preincubated with $A b$ 's 30 minutes before addition of Jurkat cells and SEE. (b) Jurkat cells were transfected with NFAT, AP-1, or NF-KB reporter plasmid and stimulated as in a. Stimulation with TPA/ionomycin was used as positive control. (c) Jurkat cells were left unstimulated, stimulated with PMA/iono, or stimulated with BJAB cells and SEE. After 16 hours, cells were stained with an R-phycoerythrin-conjugated anti-CD19 Ab and a fluorescein-conjugated anti-CD69 Ab. Jurkat cells were identified as being negative for CD19 (a B cell marker, present at the cell surface of BJAB cells). Histograms represent CD69 expression on Jurkat cells.

cells were fixed in $4 \%$ paraformaldehyde before incubation with a specific anti-Ha tag $\mathrm{Ab}$ in a buffer containing $0.02 \%$ saponin (Sigma Chemical Co.).

Jurkat cell stimulation. Jurkat cells were transiently transfected with plasmids encoding the luciferase gene under the control of multimerized copies of RE/AP, NFAT, AP-1, or NF- $\mathrm{KB}$ transcription elements. Twentyfour hours after transfection, $10^{4}$ transfected Jurkat cells were stimulated with $10^{4} \mathrm{BJAB}$ cells and SEE $(200$ $\mathrm{ng} / \mathrm{ml}$ ) in a final volume of $100 \mu \mathrm{l}$. Sixteen hours after stimulation, the cells were harvested for luciferase assays. For blocking experiments, Ab's were used at a final concentration of $10 \mu \mathrm{g} / \mathrm{ml}$ and added to the BJAB cells 30 minutes before the addition of Jurkat cells and SEE. As a positive control for transfection efficiency, transfected Jurkat cells were incubated with TPA (20 $\mathrm{ng} / \mathrm{ml}$ ) and ionomycin $(500 \mathrm{nM})$.

NK cell-mediated cytotoxicity. Cytotoxicity of NKL cell line was measured in a standard 4-hour ${ }^{51} \mathrm{Cr}$ release assay using $\mathrm{Na}_{2}{ }^{51} \mathrm{CrO}_{4}$-labeled cells as targets. Experiments were conducted in triplicate at various effectorto-target ratios. The percentage of specific ${ }^{51} \mathrm{Cr}$ release (specific lysis) was calculated according to the formula: percentage of specific lysis $=[$ (experimental release - spontaneous release)/(maximum release - spontaneous release) $] \times 100$.

\section{Results}

K5 but not $K 3$ downregulates B7-2 and ICAM-1. To address the possibility that $\mathrm{K} 3$ or $\mathrm{K} 5$ could modulate other APC components implicated in immune activation, we transduced a $B$ cell line (BJAB) with retroviral vectors expressing $\mathrm{K} 3$ or K5. Mass cultures of each transduction, representing pools of more than 100,000 colonies of primary transductants, were selected for assay. Each pool displayed strong downregulation of MHC-I and $\beta 2$-microglobulin $(\beta 2 \mathrm{~m})$, as judged by flow cytometry. (Figure 1). These cells were then assayed for surface levels of B7-1 (CD80), B7-2 (CD86), and ICAM-1 (CD54). As shown in Figure 1, K3 expression did not influence the surface distribution of any of these molecules. However, K5 expression diminished B7-2 surface expression 60- to 100-fold, while it had no impact on B7-1 surface accumulation. Cells expressing K5 displayed an equally large decrement in ICAM-1 surface staining. These decrements were specific: neither K5 (Figure 1) nor K3 (data not shown) reduced the surface levels of MHC-II, CD43, CD58, CD40, CD48, CD33, CD 22, CD20, CD19, or CD18. Thus, this downregulation is not the result of global redistribution of all polypeptides from the BJAB cell membrane. It is also not the result of $\mathrm{K} 5$ overexpression: levels of K5-specific mRNA are, in fact, lower in these lines than in lytically induced BCBL-1 cells (L. Coscoy, unpublished data). While this manuscript was under review, similar results were reported by Ishido et al. (39).

To explore the fate of the B7 and ICAM chains, we asked if the disappearance of these chains from the cell surface, like that of MHC-I and $\beta 2 \mathrm{~m}$ chains, was due to enhanced endocytosis. To do this we asked if surface levels of the downregulated proteins could be restored by coexpression of a dynamin dominant negative mutant. Dynamin is an essential participant in endocytosis, and its inhibition selectively blocks the endocytic pathway. The dynamin K44E mutation is negative for dynamin function and strongly inhibits endogenous (wild-type) dynamin function (40), making it a useful reagent for this purpose. Figure 2a shows that, in the presence of K44E, but not wild-type dynamin, surface levels of both B7-2 (CD86) and ICAM-1 (CD54) are restored to nearly normal levels. This effect is specific, since overexpres- 


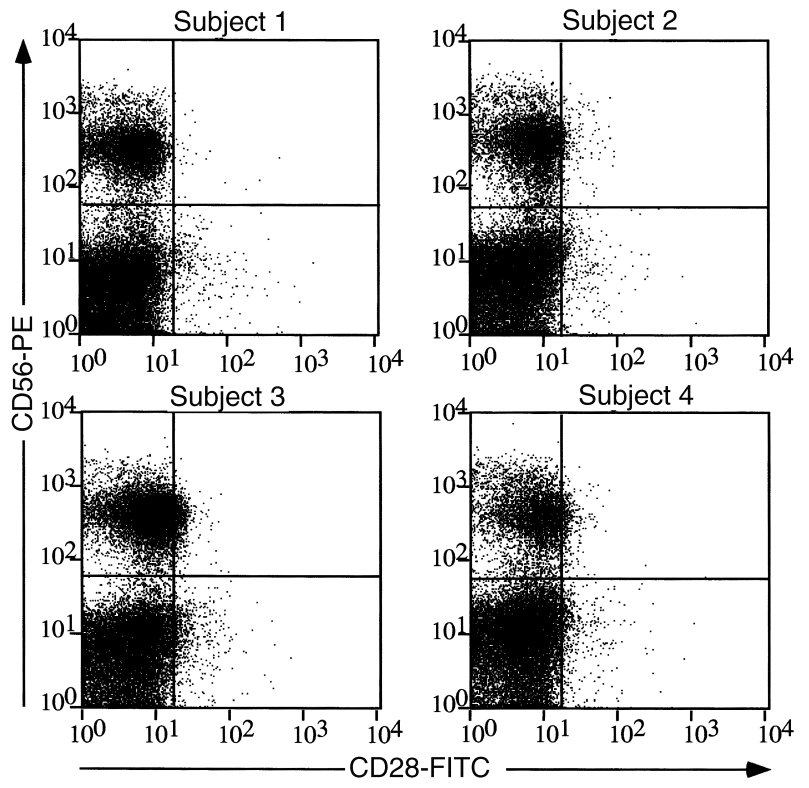

Figure 4

Human peripheral blood-derived NK cell lines do not stain for CD28. Human PBMCs were isolated from heparinized venous blood from four healthy adult volunteer donors by Ficoll-density centrifugation. Cells were stained with saturating amount of fluorescein-conjugated anti-CD28, TriColor anti-CD3, and phycoerythrin-conjugated antiCD56 Ab's and analyzed by flow cytometry. Dot plots represent the CD28 and CD56 expression status of the CD3-negative population.

sion of K44E does not affect the cell surface expression of other molecules, for example CD58 (Figure 2a).These data indicate that, as for MHC-I, the downregulatory effect of $\mathrm{K} 5$ protein is exerted chiefly through enhanced endocytosis of its ICAM and B7 targets.

What is the fate of the chains that have undergone endocytosis? Figure $2 \mathrm{~b}$ shows that the reduced levels of surface staining of both molecules is mirrored by a reduced level of intracellular accumulation of the chains, as revealed by flow-cytometric analysis in the presence of saponin. This suggests that the chains underwent degradation after internalization. This degradation can be partially relieved by exposure of the cells to ammonium chloride, which raises endolysosomal $\mathrm{pH}$ and impairs protease activity in this compartment (Figure 2b). This suggests that most newly internalized chains are degraded by endolysosomal proteases.

Functional significance of K5-mediated B7-2 and ICAM downregulation. We next tested the functional significance of ICAM-1 and B7-2 downregulation in T cell stimulation accompanying antigen presentation. Since $\mathrm{K} 5$ does not function in murine cells (ref. 35; L. Coscoy, unpublished data), we were unable to use standard in vitro assays involving antigen-specific stimulation of primary T cells from TCR-transgenic mice. Therefore, we used instead a model system that employs cultured human $\mathrm{T}$ and $\mathrm{B}$ cell lines and replaces conventional antigen with staphylococcal superantigen (41). Super- antigens mimic conventional antigens in that they lead to functional interactions of TCR with MHC molecules; however, they have the advantage of allowing recognition of multiple TCR v-regions, producing strong T cell responses. Our assay measures the ability of BJAB cells (bearing or lacking K5 genes) to stimulate Jurkat T cells in the presence of SEE. Activation is assayed by the upregulation of several transcription factors known to be stimulated by $\mathrm{T}$ cell-APC engagement, including NFAT and AP-1. Since their upregulation is resistant to CTLA4-Ig, NFAT and AP-1 activation reflect stimulatory pathways other than those mediated by B7-CD28 (42), most likely those mediated by TCR-based ITAM signaling. (These pathways, in turn, are known to be augmented by ICAM/LFA-1 interactions.) To assay for B7-CD28-dependent pathways, we took advantage of the fact that such pathways activate a composite transcription factor-binding site known as RE/AP (42). Luciferase genes under the control of the RE/AP element faithfully recapitulate $\mathrm{CD} 28$-dependent costimulation, and their expression is blocked by CTLA4- $\operatorname{Ig}(42)$. In fact, upregulation of this reporter correlates closely with enhanced IL-2 release during T cell activation (42).

Accordingly, Jurkat cells were transiently transfected with luciferase reporters under the control of either the RE/AP, NFAT, or AP-1 regulatory elements from the IL-2 promoter. Sixteen hours after transfection, these Jurkat cells were stimulated by incubation with SEE plus control BJAB cells or K5-expressing BJAB, and luciferase activity was measured 12 hours later. As shown in Figure 3a, incubation of transfected Jurkat cells with BJAB or BJAB-K5 alone without SEE was insufficient for activation of the RE/AP-dependent promoter. As expected, activation of this reporter was strongly induced by the combination of BJAB and SEE. However, this activation was greatly reduced $(60-80 \%)$ by the expression of $\mathrm{K} 5$ in the BJAB line, indicating that reduction in B7-2 strongly attenuated $\mathrm{T}$ cell activation. There was, however, a small amount

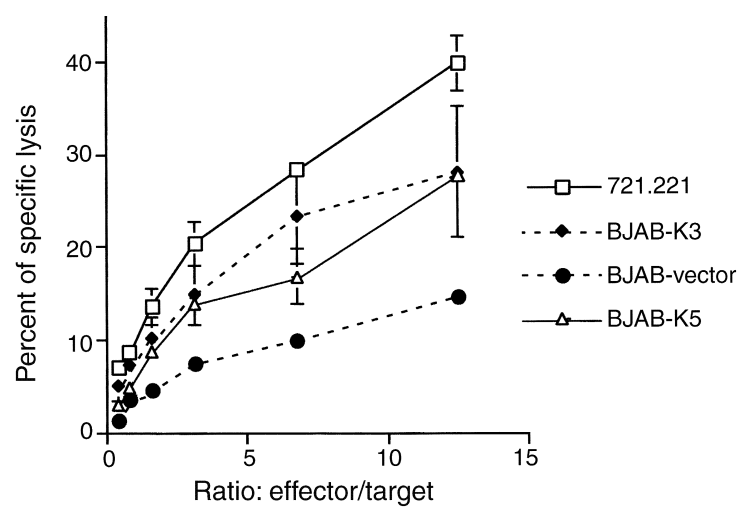

Figure 5

NKL mediate MHC-restricted cytotoxicity. Each target cell line was assessed for killing by the NKL cell line at various effector-to-target ratios (721.221 is a human $\mathrm{B}$ cell line lacking MHC-I molecules). Cytotoxicity was measured by ${ }^{51} \mathrm{Cr}$ release after 4 hours. 
of residual RE/AP activation observed in the presence of K5 (Figure 3a). Unlike primary B cells, which do not express $\mathrm{B} 7-1$ in the resting state, $\mathrm{BJAB}$ cells constitutively express both B7-1 and B7-2; we speculated that this residual activation was due to the presence of B7-1 on the BJAB cell surface. Consistent with this, this residual RE/AP activity could be ablated by incubating the BJAB-K5/Jurkat cocultures with anti-B7-1 $\mathrm{Ab}$ (Figure 3a). Thus, K5 expression effectively removes virtually all functional B7-2 from the cell surface. The significance of the remaining B7-1 will be considered in the Discussion section.

Using an identical assay format, but replacing the $\mathrm{RE} / \mathrm{AP}$ reporter in Jurkat cells with a luciferase reporter dependent upon NFAT activation, we found that substantially less NFAT activity was induced in T cells stimulated by BJAB-K5 cells than by control BJAB cells (Figure 3b). A smaller but still significant inhibition of AP-1 activation was likewise observed when K5 was expressed in the SEE-presenting BJAB cells (Figure 3b). SEE-dependent NF- $\kappa \mathrm{B}$ activation was also strongly diminished by K5 expression (Figure 3b). Thus, pathways that are not dependent upon B7-CD28 interactions are also downregulated by K5 expression. ICAMLFA interactions are known to contribute to some of these activation events $(43,44)$.

The ability of $\mathrm{K} 5$ to impair T cell activation was also assayed by examining surface expression of CD69 in the responding $\mathrm{T}$ cell. CD69 is a marker of cell activation that is prominently induced via TCR-mediated signaling. BJAB cells expressing $\mathrm{K} 5$ or a control vector were added to Jurkat cells in the presence of SEE, and the levels of CD69 expressed in Jurkat cells were examined by flow cytometry. As shown in Figure 3c, K5 expression in the APCs substantially impaired surface upregulation of CD69 in the responding T cells.

$K 5$ expression does not lead to resistance of cells to NK-mediated cytolysis. In their earlier work, Ishido et al. (39) reported that $\mathrm{K} 5$ expression reduced the susceptibility of target cells to killing mediated by the NK cell line YTS. This cell line, derived from a leukemic patient, is very unusual in that it displays expression of $\mathrm{CD} 28$ and can recognize $\mathrm{B} 7$ molecules on the target cell surface (45). Ishido et al. showed clearly that the escape from YTS killing was due to K5-mediated downregulation of B7-2 and suggested that the function of $\mathrm{K} 5$ was to promote escape from NK cell killing (to which KSHVinfected cells would otherwise be susceptible based on the reduction of surface MHC-I molecules mediated by the joint action of $\mathrm{K} 3$ and $\mathrm{K} 5)$ (33-35).

While the escape from YTS killing is clearly documented, it remains unclear whether K5's prime function in vivo is to mediate escape from NK cell killing more generally. Figure 4 shows that when the NK cell population in the circulation of four independent normal human subjects is examined by flow cytometry, cells bearing NK markers (CD56) lack evidence of CD28 expression. Indeed, similar findings have been observed previously in cultured primary human NK cell lines expanded from the PBMC compartment (46). To determine the functional consequences of $\mathrm{K} 5$ expression for NK cell-mediated cytotoxicity, we employed a more representative NK cell line, NKL, in a killing assay. Like primary NK cells, NKL cells do not express surface CD28 (47). As expected, NKL cells efficiently killed control BJAB cells expressing the empty pcDNA3 vector (Figure 5). More importantly, however, $\mathrm{BJAB}$ cells engineered to express $\mathrm{K} 5$ not only displayed susceptibility to NKL killing but were, in fact, more sensitive to this killing than the controls (Figure 5). This result is in accordance with the diminished levels of MHC-I on such cells that result from the K5-stimulated endocytosis of class I chains $(33,34)$. While we do not observe strong effects of $\mathrm{K} 5$ on protection from NK-mediated cytolysis, we acknowledge that the paucity of cultured NK lines and their uncertain relationship to in vivo NK cells makes it difficult to entirely exclude such effects. Whatever their role in NK biology may be, the best-established functions of B7-2 and ICAM-1 are in the pathway of $\mathrm{T}$ cell costimulation, and our data strongly support a major role for $\mathrm{K} 5$ in the modulation of this pathway (see below).

\section{Discussion}

We report here, in agreement with Ishido et al. (39), that the KSHV K5 (but not K3) protein downregulates ICAM-1 and B7-2 from the cell surface of B cells by inducing their endocytosis and degradation. This downregulation is strong enough to impair T cell stimulation induced by $\mathrm{B}$ cell-T cell interactions during antigen presentation. To our knowledge, this is the first report describing a viral protein that modulates the process of $\mathrm{T}$ cell costimulation.

We were initially surprised that a viral blockade of costimulation would be focused selectively on B7-2 rather than attacking both B7-1 and B7-2. Many functions of these two proteins are redundant, a result strongly attested to by the fact that in knockout mice lesions affecting these loci singly have a much less dramatic phenotype than does the double knockout (48). However, in such animals immune responses involve many types of APCs, including dendritic cells and macrophages as well as B cells. Of those cell types, only $B$ cells are efficiently infected by KSHV in vivo.

In contrast to naive $B$ cells, it is now accepted that activated B cells are potent APCs (49-52). This difference has generally been attributed to the induction of B7 costimulatory molecules, which are absent on naive $B$ cells by $B$ cell activation. Activation of $B$ cells (e.g., via CD40-CD40L signaling resulting from interaction with activated $T$ cells) leads to efficient upregulation of surface B7-2 within 4 hours. By contrast, upregulation of B7-1 upon stimulation appears to be much more modest and occurs substantially later (53). B cells can also be activated by signals other than those provided by activated $T$ cells, for example, cross-linking of $B$ cell antigen receptors by anti-Ig Ab's and/or antigen (51, $52,54)$ or exposure to bacterial LPS. In several recent 
studies $(52,55,56)$ it has been reported that B cells activated by soluble antigen exposure can even prime naive $\mathrm{T}$ cells. This priming appears to depend solely upon $\mathrm{B} 7-2$, because $\mathrm{B} 7-1$ is not expressed by $\mathrm{B}$ cells in vivo in this context $(52,57)$. Thus, it appears that B7-2 plays a strong role in the antigen-presenting behavior of primary B cells in vivo. Moreover, we note that K5 expression is part of the lytic replicative cycle of KSHV. The herpesviral lytic cycle is strongly cytopathic, leading to inhibition of host cell macromolecular synthesis and host cell death as viral replication progresses. Such conditions would be expected to interfere with the late upregulation of B7-1.

ICAM-1 is thought to function to promote immune synapse formation (25-27) and promote sustained TCR signaling $(17,18)$. Accordingly, ICAM-1 downregulation would be expected to contribute to impaired $\mathrm{T}$ cell activation and TCR signaling. While it is difficult to rigorously quantitate the contribution of ICAM-1 blockade to the impairment of $\mathrm{T}$ cell activation observed here, the fact that $\mathrm{T}$ cell signaling pathways not ablated by CTLA4-Ig were impaired by K 5 expression in the APCs suggests that these effects were not the result of the B7-2 downregulation and were likely due, at least in part, to ICAM-1 modulation.

We propose that downregulation of proteins associated with immune synapse formation and costimulation may reduce the visibility of KSHV-infected B-cells to $\mathrm{T}$ helper cells, thereby diminishing $\mathrm{CD}^{+} \mathrm{T}$ cell-mediated cytolysis, antiviral cytokine production, and upregulation of stimulatory signals for CTL generation. We think it likely that this effect is most important early in the natural history of KSHV infection, when the number of infected cells is small and the levels of soluble viral particles and antigens are low. These conditions maximize dependence upon costimulatory signals; also, at this point in infection most viral antigens will be confined to infected B cells. Diminished T cell recognition of such cells would result in reduced antiviral cytokine responses and impaired production of stimulatory signals for CTL generation, thereby allowing the virus to establish a foothold in the infected host.

1. Unanue, E.R. 1984. Antigen-presenting function of the macrophage. Annu. Rev. Immunol. 2:395-428.

2. Valitutti, S., Dessing, M., Aktories, K., Gallati, H., and Lanzavecchia, A. 1995. Sustained signaling leading to T cell activation results from prolonged T cell receptor occupancy. Role of T cell actin cytoskeleton. J. Exp. Med. 181:577-584.

3. Iezzi, G., Karjalainen, K., and Lanzavecchia, A. 1998. The duration of antigenic stimulation determines the fate of naive and effector $\mathrm{T}$ cells. Immunity. 8:89-95.

4. Demotz, S., Grey, H.M., and Sette, A. 1990. The minimal number of class II MHC-antigen complexes needed for $\mathrm{T}$ cell activation. Science. 249:1028-1030.

5. Harding, C.V., and Unanue, E.R. 1990. Quantitation of antigen-presenting cell MHC class II/peptide complexes necessary for T-cell stimulation. Nature. 346:574-576.

6. Brower, R.C., et al. 1994. Minimal requirements for peptide mediated activation of CD8+ CTL. Mol. Immunol. 31:1285-1293.

7. Sykulev, Y., Joo, M., Vturina, I., Tsomides, T.J., and Eisen, H.N. 1996. Evidence that a single peptide-MHC complex on a target cell can elicit a cytolytic $\mathrm{T}$ cell response. Immunity. 4:565-571.

8. Delon, J., Bercovici, N., Raposo, G., Liblau, R., and Trautmann, A. 1998. Antigen-dependent and -independent $\mathrm{Ca} 2+$ responses triggered in $\mathrm{T}$ cells by dendritic cells compared with B cells. J. Exp. Med. 188:1473-1484.

9. Shaw, A.S., and Dustin, M.L. 1997. Making the T cell receptor go the distance: a topological view of T cell activation. Immunity. 6:361-369.

10. Springer, T.A. 1990. Adhesion receptors of the immune system. Nature. 346:425-434.

11. Greenfield, E.A., Nguyen, K.A., and Kuchroo, V.K. 1998. CD28/B7 costimulation: a review. Crit. Rev. Immunol. 18:389-418.

12. Lenschow, D.J., Walunas, T.L., and Bluestone, J.A. 1996. CD28/B7 system of T cell costimulation. Annu. Rev. Immunol. 14:233-258.

13. Chambers, C.A., and Allison, J.P. 1999. Costimulatory regulation of T cell function. Curr. Opin. Cell Biol. 11:203-210.

14. Blair, P.J., et al. 1997. CD28 co-receptor signal transduction in T-cell activation. Biochem. Soc. Trans. 25:651-657.

15. Sperling, A.I., and Bluestone, J.A. 1996. The complexities of T-cell costimulation: CD28 and beyond. Immunol. Rev. 153:155-182.

16. Dustin, M.L., and Springer, T.A. 1989. T-cell receptor cross-linking transiently stimulates adhesiveness through LFA-1. Nature. 341:619-624.

17. Van Kooyk, Y., and Figdor, C.G. 1997. Signalling and adhesive properties of the integrin leucocyte function-associated antigen 1 (LFA-1). Biochem. Soc. Trans. 25:515-520.

18. Stewart, M., and Hogg, N. 1996. Regulation of leukocyte integrin function: affinity vs. avidity. J. Cell. Biochem. 61:554-561.

19. Bachmann, M.F., et al. 1997. Distinct roles for LFA-1 and CD28 during activation of naive T cells: adhesion versus costimulation. Immunity. 7:549-557.

20. Dubey, C., Croft, M., and Swain, S.L. 1995. Costimulatory requirements of naive CD4+ T cells. ICAM-1 or B7-1 can costimulate naive CD4 T cell activation but both are required for optimum response. J. Immunol. 155:45-57.

21. Sedwick, C.E., et al. 1999. TCR, LFA-1, and CD28 play unique and complementary roles in signaling $\mathrm{T}$ cell cytoskeletal reorganization. $J$. Immunol. 162:1367-1375.

22. Paul, W.E., and Seder, R.A. 1994. Lymphocyte responses and cytokines. Cell. 76:241-251.

23. Dustin, M.L., et al. 1998. A novel adaptor protein orchestrates receptor patterning and cytoskeletal polarity in T-cell contacts. Cell. 94:667-677.

24. Wulfing, C., Sjaastad, M.D., and Davis, M.M. 1998. Visualizing the dynamics of $T$ cell activation: intracellular adhesion molecule 1 migrates rapidly to the $\mathrm{T}$ cell/B cell interface and acts to sustain calcium levels. Proc. Natl. Acad. Sci. USA. 95:6302-6307.

25. Wulfing, C., and Davis, M.M. 1998. A receptor/cytoskeletal movement triggered by costimulation during $\mathrm{T}$ cell activation. Science. 282:2266-2269.

26. Grakoui A, et al. 1999. The immunological synapse: a molecular machine controlling T cell activation. Science. 285:221-227.

27. Monks, C.R., Freiberg, B.A., Kupfer, H., Sciaky, N., and Kupfer, A. 1998. Three-dimensional segregation of supramolecular activation clusters in T cells. Nature. 395:82-86.

28. Tortorella, D., Gewurz, B.E., Furman, M.H., Schust, D.J., and Ploegh, H.L. 2000. Viral subversion of the immune system. Annu. Rev. Immunol. 18:861-926.

29. Hengel, H., and Koszinowski, U.H. 1997. Interference with antigen processing by viruses. Curr. Opin. Immunol. 9:470-476.

30. Ploegh, H.L. 1998. Viral strategies of immune evasion. Science. 280:248-253.

31. Hengel, H., et al. 1999. Cytomegaloviral control of MHC class I function in the mouse. Immunol. Rev. 168:167-176.

32. Yewdell, J.W., and Bennink, J.R. 1999. Mechanisms of viral interference with MHC class I antigen processing and presentation. Annu. Rev. Cell Dev. Biol. 15:579-606.

33. Coscoy, L., and Ganem, D. 2000. Kaposi's sarcoma-associated herpesvirus encodes two proteins that block cell surface display of MHC class I chains by enhancing their endocytosis. Proc. Natl. Acad. Sci. USA. 97:8051-8056.

34. Ishido, S., Wang, C., Lee, B.S., Cohen, G.B., and Jung, J.U. 2000. Downregulation of major histocompatibility complex class I molecules by Kaposi's sarcoma-associated herpesvirus K3 and K5 proteins. J. Virol. 74:5300-5309.

35. Stevenson, P.G., Efstathiou, S., Doherty, P.C., and Lehner, P.J. 2000. Inhibition of MHC class I-restricted antigen presentation by gamma 2-herpesviruses. Proc. Natl. Acad. Sci. USA. 97:8455-8460.

36. Whitby, D., and Boshoff, C. 1998. Kaposi's sarcoma herpesvirus as a new paradigm for virus-induced oncogenesis. Curr. Opin. Oncol. 10:405-412.

37. Haque, M., et al. 2001. Major histocompatibility complex class I molecules are down-regulated at the cell surface by the $\mathrm{K} 5$ protein encoded by Kaposi's sarcoma-associated herpesvirus/human herpesvirus-8. J. Gen. Virol. 82:1175-1180.

38. Ambroziak, J.A., et al. 1995. Herpes-like sequences in HIV-infected and uninfected Kaposi's sarcoma patients. Science. 268:582-583.

39. Ishido, S., et al. 2000. Inhibition of natural killer cell-mediated cytotoxicity by Kaposi's sarcoma-associated herpesvirus K5 protein. Immunity. 13:365-374. 
40. Herskovits, J.S., Burgess, C.C., Obar, R.A., and Vallee, R.B. 1993. Effects of mutant rat dynamin on endocytosis. J. Cell. Biol. 122:565-578.

41. Fraser, J.D., Newton, M.E., and Weiss, A. 1992. CD28 and T cell antigen receptor signal transduction coordinately regulate interleukin 2 gene expression in response to superantigen stimulation. J. Exp. Med. 175:1131-1134.

42. Shapiro, V.S., Mollenauer, M.N., Greene, W.C., and Weiss, A. 1996. c-rel regulation of IL-2 gene expression may be mediated through activation of AP-1. J. Exp. Med. 184:1663-1669.

43. Lub, M., van Kooyk, Y., and Figdor, C.G. 1995. Ins and outs of LFA-1. Immunol. Today. 16:479-483.

44. Van Seventer, G.A., et al. 1992. Costimulation of T cell receptor/CD3mediated activation of resting human CD4+ T cells by leukocyte function-associated antigen-1 ligand intercellular cell adhesion molecule- 1 involves prolonged inositol phospholipid hydrolysis and sustained increase of intracellular Ca2+ levels. J. Immunol. 149:3872-3880.

45. Azuma, M., Cayabyab, M., Buck, D., Phillips, J.H., and Lanier, L.L. 1992. Involvement of CD28 in MHC-unrestricted cytotoxicity mediated by a human natural killer leukemia cell line. J. Immunol. 149:1115-1123.

46. Nagler, A., Lanier, L.L., Cwirla, S., and Phillips, J.H. 1989. Comparative studies of human FcRIII-positive and negative natural killer cells. $J$. Immunol. 143:3183-3191.

47. Robertson, M.J., et al. 1996. Characterization of a cell line, NKL, derived from an aggressive human natural killer cell leukemia. Exp. Hematol. 24:406-415.

48. Borriello, F., et al. 1997. B7-1 and B7-2 have overlapping, critical roles in immunoglobulin class switching and germinal center formation. Immunity. 6:303-313.

49. Ron, Y., and Sprent, J. 1987. T cell priming in vivo: a major role for B cells in presenting antigen to $\mathrm{T}$ cells in lymph nodes. J. Immunol. 138:2848-2856.

50. Kurt-Jones, E.A., et al. 1988. The role of antigen-presenting B cells in T cell priming in vivo. Studies of B cell-deficient mice. J. Immunol. 140:3773-3778

51. Morris, S.C., Lees, A., and Finkelman, F.D. 1994. In vivo activation of naive T cells by antigen-presenting B cells. J. Immunol. 152:3777-3785.

52. Constant, S.L. 1999. B lymphocytes as antigen-presenting cells for CD4+ $\mathrm{T}$ cell priming in vivo. J. Immunol. 162:5695-5703.

53. Evans, D.E., Munks, M.W., Purkerson, J.M., and Parker, D.C. 2000. Resting B lymphocytes as APC for naive T lymphocytes: dependence on CD40 ligand/CD40. J. Immunol. 164:688-697.

54. Eynon, E.E., and Parker, D.C. 1993. Parameters of tolerance induction by antigen targeted to B lymphocytes. J. Immunol. 151:2958-2964.

55. Constant, S., Schweitzer, N., West, J., Ranney, P., and Bottomly, K. 1995 B lymphocytes can be competent antigen-presenting cells for priming CD4+ T cells to protein antigens in vivo. J. Immunol. 155:3734-3741.

56. Constant, S., et al. 1995. Peptide and protein antigens require distinct antigen-presenting cell subsets for the priming of CD4+ T cells. $J$. Immunol. 154:4915-4923.

57. Lenschow, D.J., et al. 1994. Differential up-regulation of the B7-1 and B7-2 costimulatory molecules after Ig receptor engagement by antigen. J. Immunol. 153:1990-1997. 